\title{
Design, Implementation and Performance Analysis of Tabu Search Based PID Controller
}

\author{
Faizullah Mahar ${ }^{1, *}$, Muhammad Amin ${ }^{2}$ \\ ${ }^{1}$ Faculty of Electrical Engineering, Balochistan University of Engineering \&Technology, Pakistan \\ ${ }^{2}$ Vice Chancellor, Balochistan University of Engineering \&Technology, Pakistan
}

Copyright $\mathrm{O} 2016$ by authors, all rights reserved. Authors agree that this article remains permanently open access under the terms of the Creative Commons Attribution License 4.0 International License

\begin{abstract}
This paper deals with design, implementation and the performance analysis of Tabu search (TS) algorithm based PID controller. The experimental results demonstrate that implantation of TS-based controller designed by the suggested technique can deliver the finest system response as compared to the conventional techniques. The TS algorithm based design of controller using the suggested technique could be used to produce a software package for engineers to achieve the optimized controller design. This paper presents the optimized PID controller design for the system using the TS algorithm. The resulting output responses using the TS design is superior to that of the conventional method can also be used to support the simulation effects. These effects demonstrate that the suggested design method is very suitable for engineers. Moreover, the suggested technique can provide the best output performance with the stable operation confirmation.
\end{abstract}

Keywords Tabu Search Algorithm, Optimization, Robustness, Performance and Controllers

\section{Introduction}

Fundamentally, the function of a controller is to generate appropriate control signals for exhibiting desired response of any physical system. Several tuning methodologies have been proposed for optimal tuning of PID controller. Since, classical tuning methods failed to optimize the parameters for the non-minimum phase systems. Hence, newly introduced algorithms which have the features of easy implementation, short execution time and robust mechanism of escaping from local optimum, are a promising tool for engineering applications [1].

The Proportional-Integral-Derivative (PID) controller has been proved the most popular controller of this century for its remarkable effectiveness, easiness of implementation and vast applicability. However, it is also hard to tune the PID controller. A number of tuning methods are done manually which are difficult and time-consuming. For using PID controller efficiently, the optimal tuning of its parameters has become a significant research area [2].

The task of controlled systems design has to respect a number of desires imposed on behavior of the system to be controlled. PID parameter optimization is a crucial constituent of several areas of research; many approaches exist to achieve an optimal behavior of the system [3].

PID Controller often includes several searched parameters and constraints that optimization are complicated and badly behaved [4]. The more motivating way for solving the optimization problem is heuristic algorithms, the texts reflect the fame of PID controller as more than hundreds of research papers embark upon different facets of it [5].

PID controller consists of Proportional, Integral and Derivative gains. The literature confirms that more than $95 \%$ of the controllers used in industries are PID type [6]. Undesirably, tuning of gains of these controllers for attaining and performance terms and robustness is tough task [7].

The prime enticement of the paper is to provide a comprehensive usage on design and implementation of Tabu based controller. Moreover, this also indulges the analysis of system robustness performance.

The remaining paper ordered as: An-overview of the $\mathrm{H} \infty$ control design problem is given in section II, TS has been explained in section III; section IV discusses the experimental results. TS-based design procedure, section V presents results of simulation, performance of the analysis, finally the conclusion is given in section VI.

\section{The $\boldsymbol{H}_{\infty}$ Control Design Problem}

Consider a system $P(s)$ of Fig.1, with inputs $w$, outputs $z$, measurement $y$, control $u$ and controller $K(s)$ if $P(\mathrm{~s})$ is being used to formulate a design problem, then it will also include weighting functions [8]. 


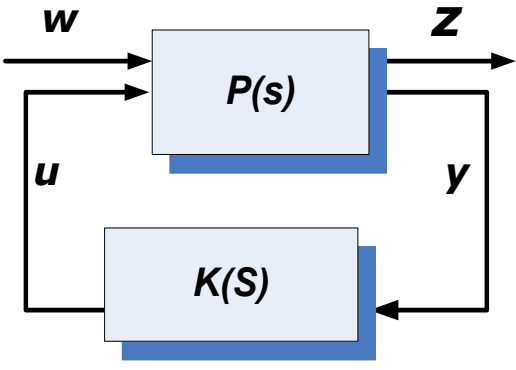

Figure 1. General $H \infty$ Configuration [8]

To minimizing the infinity-norm of the transfer function from $w$ to $z, P(s)$ can be partitioned as [9]:

$$
P(s)=\left[\begin{array}{ll}
P_{11}(s) & P_{12}(s) \\
P_{21}(s) & P_{22}(s)
\end{array}\right]
$$

The closed loop transfer function from $w$ to $z$ can be obtained directly as:

$$
Z=F_{l}(P, K) w
$$

Where $F_{l}(P, K)=P_{11}+P_{12} K\left(I-P_{22} K\right)^{-1} P_{21}$ is called the lower fractional transformation of $P$ and $K$. Therefore, the optimal $H_{\infty}$ control problem is to minimize the $H_{\infty}$ norm of $F_{l}(P, K)$, i.e, $\left\|F_{l}(P, K)\right\|_{\infty}$

\section{A. Weight Selection}

For selecting the weights some guide lines available in [10]. The weighting functions are chosen as Eq. (3):

$$
W_{1}=K \frac{s+\alpha}{s+\beta}
$$

Where $K, \alpha, \beta$ are positive numbers, $\beta$ is selected as small number for integral action. The lowest achievable value of gamma and corresponding maximum stability margin are calculated by:

$$
\gamma=(\varepsilon)^{-1}=\sqrt{\left(1+\lambda_{\max }(X Z)\right.}
$$

Where $\lambda_{\max }$ represent maximum Eigen value, $\mathrm{X}$ and $\mathrm{Z}$ are the solution to the algebraic Riccati equation.

Glover and Doyle derived necessary and sufficient conditions for the existence of an $H_{\infty}$ suboptimal solution and further parameterized all such controllers results are obtained on following assumption [11-12].

$$
\begin{aligned}
& \text { i. } \quad\left(\begin{array}{lll}
A_{1} & B_{2} & C_{2}
\end{array}\right) \text { is stabilization and detectable } \\
& \text { ii. } \quad D_{12} \text { and } D_{21} \text { have full rank } \\
& \text { iii. } \quad\left[\begin{array}{cc}
A-j w I & B_{2} \\
C_{1} & D_{12}
\end{array}\right] \text { have full column rank for all } w \\
& \text { iv. } \quad\left[\begin{array}{cc}
A-j w I & B_{1} \\
C_{2} & D_{21}
\end{array}\right] \text { have full row rank for all } w \\
& \text { v. } D_{11}=0 \text { and } D_{22}=0
\end{aligned}
$$

The following plant model of the plant is taken from [2].

$$
G(s)=\frac{551.3 e^{(-0.12 s)}}{\left(s^{2}+43.26 s+536.9\right)}
$$

The $p$ controller structure is taken as vector $p$ of the controller parameters is given by the $p=\left[\begin{array}{lll}\mathrm{Kp} & K_{i} & K_{d}\end{array}\right]$

From ref. [4] the Controller $K(p)$ can be written as:

$$
K(p)=W_{1} K_{\infty} W_{2}
$$

It is assumed that $W_{1}$ is invertible and $W_{2}=1$

$$
K_{\infty}=W_{1}^{-1} K(p)
$$

By substituting Eq. (10) into Eq. (4), we have

$$
J_{\cos t}=\|\left[\begin{array}{c}
I \\
W^{-1} K(p)
\end{array}\right]\left(I+G_{S} W^{-1} K(p)^{-1}\left(\begin{array}{ll}
I & \left.G_{S}\right)
\end{array} \|_{\infty}\right.\right.
$$

\subsection{Statement of the Problem}

Controllers often include many searched parameters and their different constraints the search optimization problem may be complicated. As shown in figure. 1 the controller take information concerning the plant. Where $y$ is controlled variable, $u$ is the control value $r$ reference and $e$ is the control error. Fortunately, a properly designed controller prevents the system from operating in unstable mode. Therefore, it is very important that the controller be engineered that will internally stabilize the plant if it is not stable primarily and satisfy certain performance level.

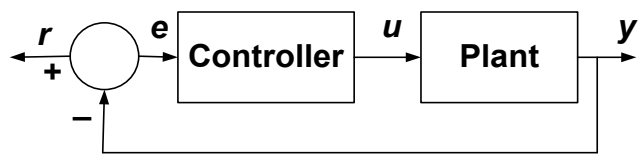

Figure 2. Simple feedback closed loop

\section{Tabu Search an Overview}

TS algorithm stands high-level heuristic algorithm for solving optimization problems. It is expansion methods based on iterations that start from any initial solution and endeavor to find out improved result. TS were proposed in its present form a few years ago by Glover [3, 7].

Currently, it has grown to be a recognized optimization approach that is quickly increasing to numerous fields of engineering and sciences. Together with other heuristic search algorithms TS has been singled out as "extremely promising" for the future treatment of practical applications [8]. Usually, TS is categorized by its capability to avoid entrapment in local optimum result and prevent cycling by the use flexible memory of search history [9].

\subsection{PID Controller Background}

PID controller consists of Proportional, Integral and Derivative gains. The structure of PID controllers is very 
simple and it works in a closed-loop system as given in Fig.6; the controller operates on the error signal that is the difference between the desired output and the actual output, and generates the actuating signal $(u)$ that drives the plant. The output of a PID controller, equal to the control input to the plant, in the time-domain is as given in Eq. (9)

$$
u(t)=K_{p} e(t)+K_{i} \int e(t) d t+K_{d} \frac{d e}{d t}
$$

The transfer function of a PID controller is found by taking the Laplace transform of Eq. (10).

$$
K_{p}+\frac{K_{i}}{s}+K_{d} s=\frac{K_{d} s^{2}+K_{p} s+K_{i}}{s}
$$

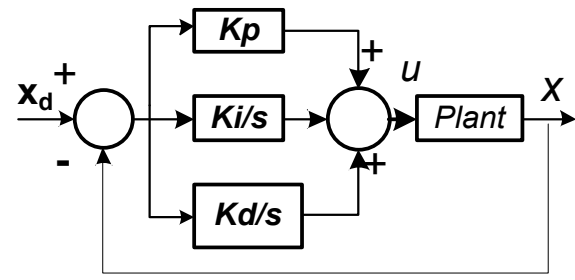

Figure 3. Structure of a SISO-PID controller

\subsection{Ts -Based Robust Controller Design}

1. Choose an initial solution $x$

2. Find a subset of $n(x)$ the neighbors of $x$ which are not in the Tabu list.

3. Find the best one ( $\left.\mathrm{x}^{\prime}\right)$ in set $n(x)$.

4. If $f\left(x^{\prime}\right)>f(x)$ then set $x^{\prime}=x^{\prime}$.

5. Modify the Tabu list.
6. If a stopping condition is met then stop, else go to the second step

\subsection{Implementation of Tabu Search}

Mutation

With probability $p_{w}$, randomly pick neighbor

With probability $1-p_{w}$, Tabu search

Tabu search

Tabu list

List of $\left\{v_{i}, c_{j}\right\}$

Tabu tenure (the length of the Tabu list)

$\mathrm{L}=\mathrm{a} * \mathrm{n}_{\mathrm{c}}+\operatorname{random}(\mathrm{g})$

$\mathrm{n}_{\mathrm{c}}$ : number of conflicted nodes

a,g: empirical parameters

\subsection{Loop Shaping}

Two steps are involved in this procedure. In first step two weighting functions $W_{1}$ and $W_{2}$ are specified to shape the original plant. The singular values of the shaped plant satisfy the closed-loop performance requirements [15].

$$
G_{S}=W_{1} G_{0} W_{2}
$$

The weighting functions are chosen as:

$$
W_{1}=\frac{0.7 s+4.0}{s+0.0001} \quad W_{2}=I
$$

Where $I$ is the identity matrix, with these weighting functions band width of the desired control system is increased from $3.4 \mathrm{rad} / \mathrm{sec}$ to $4.2 \mathrm{rad} / \mathrm{sec}$, drastically improves the performance and robustness

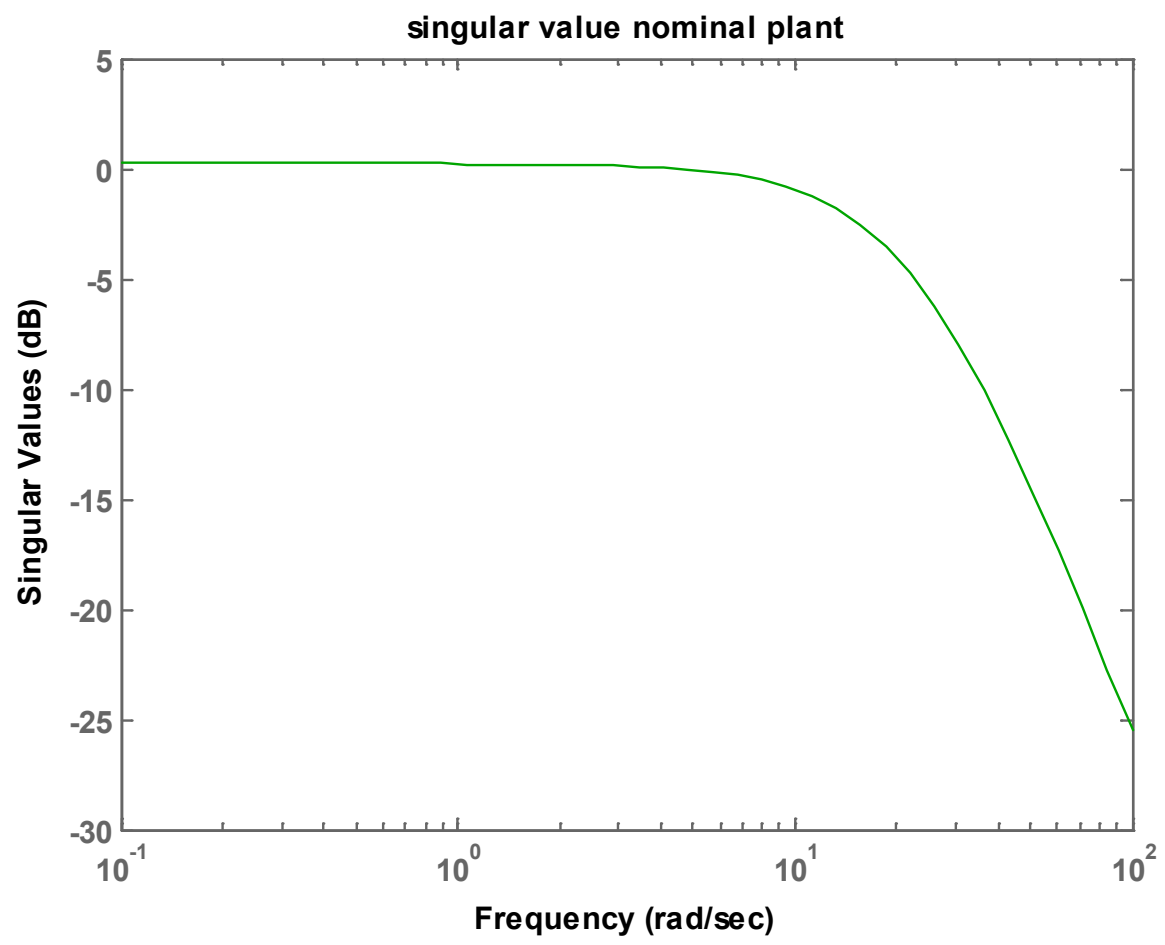

Figure 4. Singular value of nominal plant 


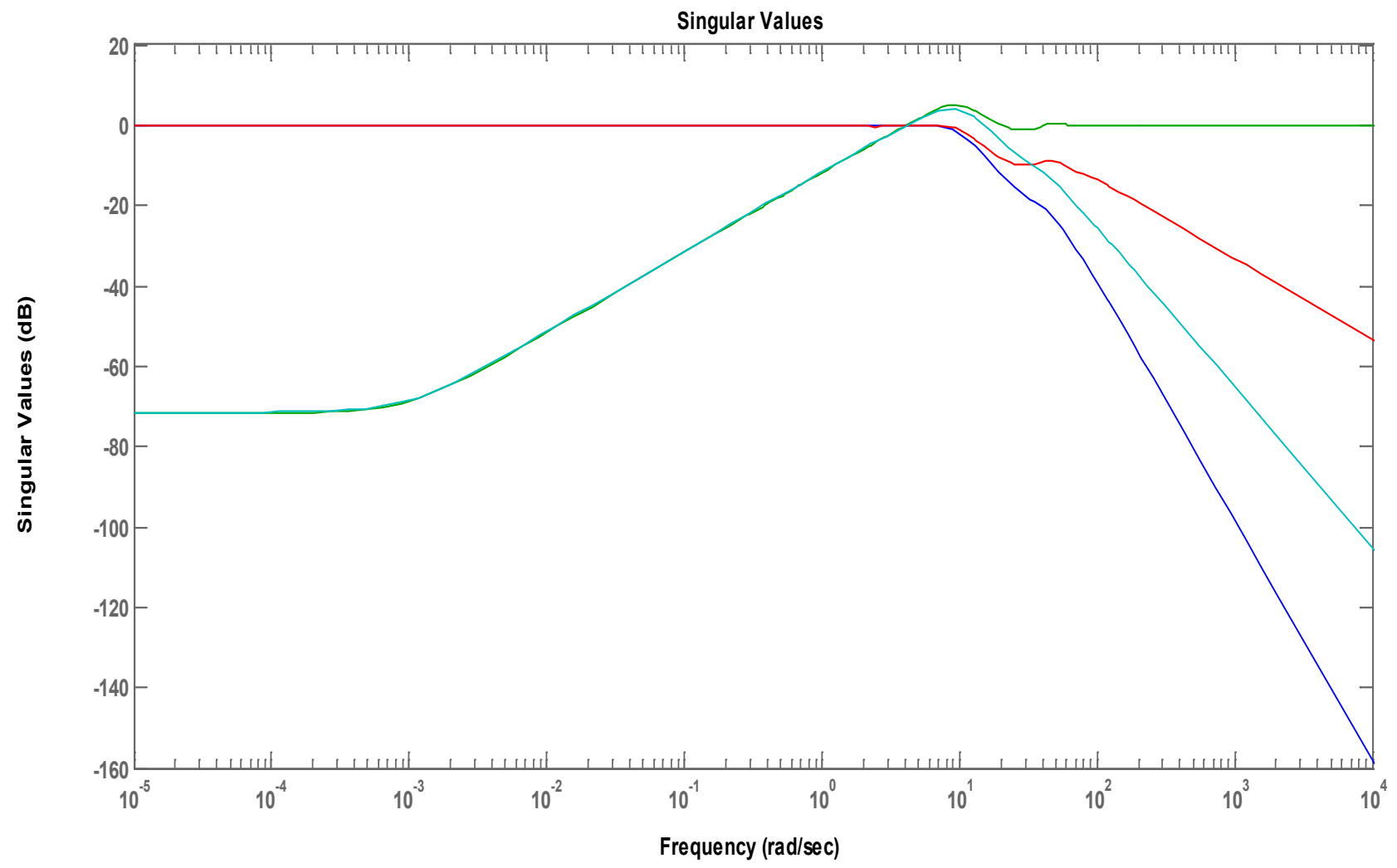

Figure 5. Singular Value of shaped plant

\section{Experimental Results}

A TS algorithm based controller has been designed for the transfer function of plant is presented by Eq. (11).

$$
G(s)=\frac{551.3 e^{(-0.12 s)}}{\left(s^{2}+43.26 s+536.9\right)}
$$

The weighs preferred are:

$$
W_{1}(s)=\frac{0.69 s+3.66}{s+0.0001}
$$

Where second weight $W_{2}$ is preferred as an identity matrix, with the help of preferred weights shaped- plant is formed as:

$$
G_{S}(s)=\frac{413.5 s+3105 e^{-0.11 s}}{s^{3}+43.26 s^{2}+356.9 s+0.4589}
$$

The $\mathrm{K}_{\infty}$ controller acquired by the help of $\mathrm{H} \infty$ loop shaping

$$
K_{\infty}(s)=\frac{512.61 s+324.2}{s^{3}+43.36 s^{2}+636.9 s+0.6609}
$$

And the controller $K_{f}(s)$ has achieved eq (15):

$$
K_{f}(s)=\frac{411.3 s^{2}+406 s+9231}{s^{5}+44.26 s^{3}+567 s^{2}+1.774 s+0.0509}
$$

The controller tuned by traditional methods given in Eq. (15) has complex structure and has $5^{\text {th }}$ order as well, observably, it would be challenging to implement for industrial control. Hence, the benefit of fixed order controller design could be achieved from recommended methodology.

The Matlab-based simulations were conceded by running the TS algorithm. The size of initial population was 100; TS algorithm converged on $51^{\text {th }}$ iteration and presented optimized CF of 1.966. 


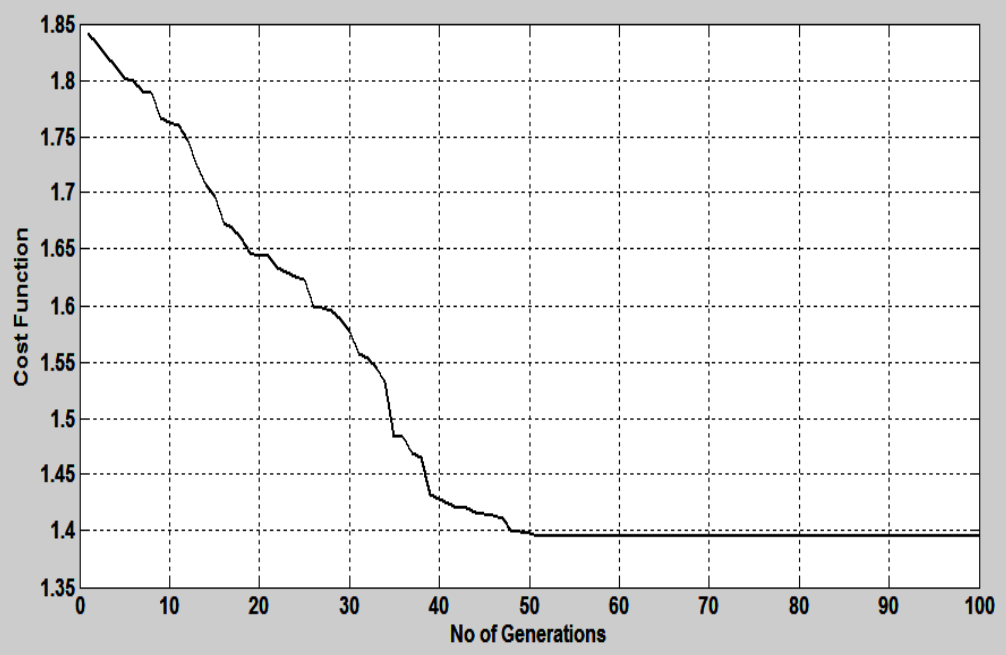

Figure 6. Convergence of TS algorithm iterations Vs CF

The optimized specific controller gains were acquired on $47^{\text {th }}$ iteration of TS algorithm and ensures gratified stability margin of 0.501 , which demonstrate that TS can find optimized result after $47^{\text {th }}$ iterations. The optimized values of controller gains are given in eq. (16).

$$
K(p)^{*}=0.796+\frac{0.779}{s}+100
$$

The time response of the system determined by optimized controller gain by using TS algorithm is depicted in fig. 2, the time response analysis extant 0.99 seconds rise time, settling time 1.33 seconds $9 \%$ overshoot.

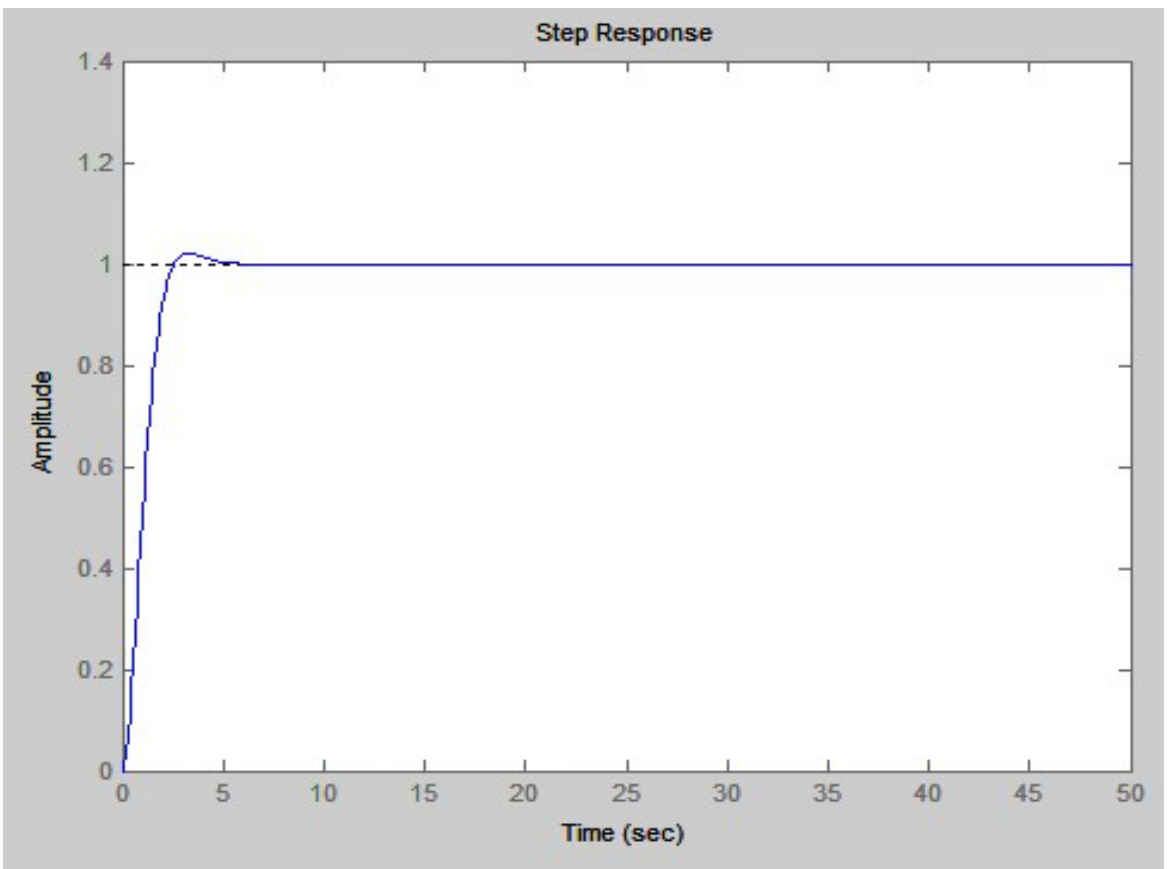

Figure 7. Time response of nominal plant with TS-based controller 
Table 1. Comparison of results between conventional techniques and TS

\begin{tabular}{|c|c|c|c|}
\hline Parameters & TS & Z-N & Hळ \\
\hline $\mathrm{Kp}$ & 0.796 & 5.44 & - \\
\hline $\mathrm{Ki}$ & 0.779 & 12.0 & - \\
\hline $\mathrm{Kd}$ & 100 & 46 & - \\
\hline $\mathrm{CF}$ & 1.37 & 3.36 & 1.45 \\
\hline
\end{tabular}

The values CF and the gains of the optimized TS-based controller were compared to $\mathrm{CF}$ values and gain obtained by traditional procedures: Z-N and $\boldsymbol{H}_{\infty}$.

The obtained results are tabulated in Table-1, which describes that TS algorithm presented much better solutions than conventional $\mathrm{Z}-\mathrm{N}$ and $\boldsymbol{H}_{\infty}$.

The PID gains obtained by using Z-N method are quite high values as compared with TS. Moreover, the best CF value is obtained from TS as compared with $H_{\infty}$

Table 2. Comparison of results between conventional techniques and TS

\begin{tabular}{|c|c|c|c|}
\hline & TS & Z-N & Hळ \\
\hline Rise time & $0.3 \mathrm{sec}$ & $3.33 \mathrm{sec}$ & $22 \mathrm{sec}$ \\
\hline Settling time & $0.16 \mathrm{sec}$ & $5 \mathrm{sec}$ & $1.2 \mathrm{sec}$ \\
\hline$\%$ overshoot & $3 \%$ & $13 \%$ & $9 \%$ \\
\hline delay time & $0.89 \mathrm{sec}$ & $3.4 \mathrm{sec}$ & $0.23 \mathrm{sec}$ \\
\hline
\end{tabular}

The above Table- 2 compares the results obtained by different techniques Z-N, $H_{\infty}$ and TS. Therefore, tuned TS based PID controller for given plant has providing much better response than the controller tuned by Z-N and $H_{\infty}$.

\section{Robustness Analysis}

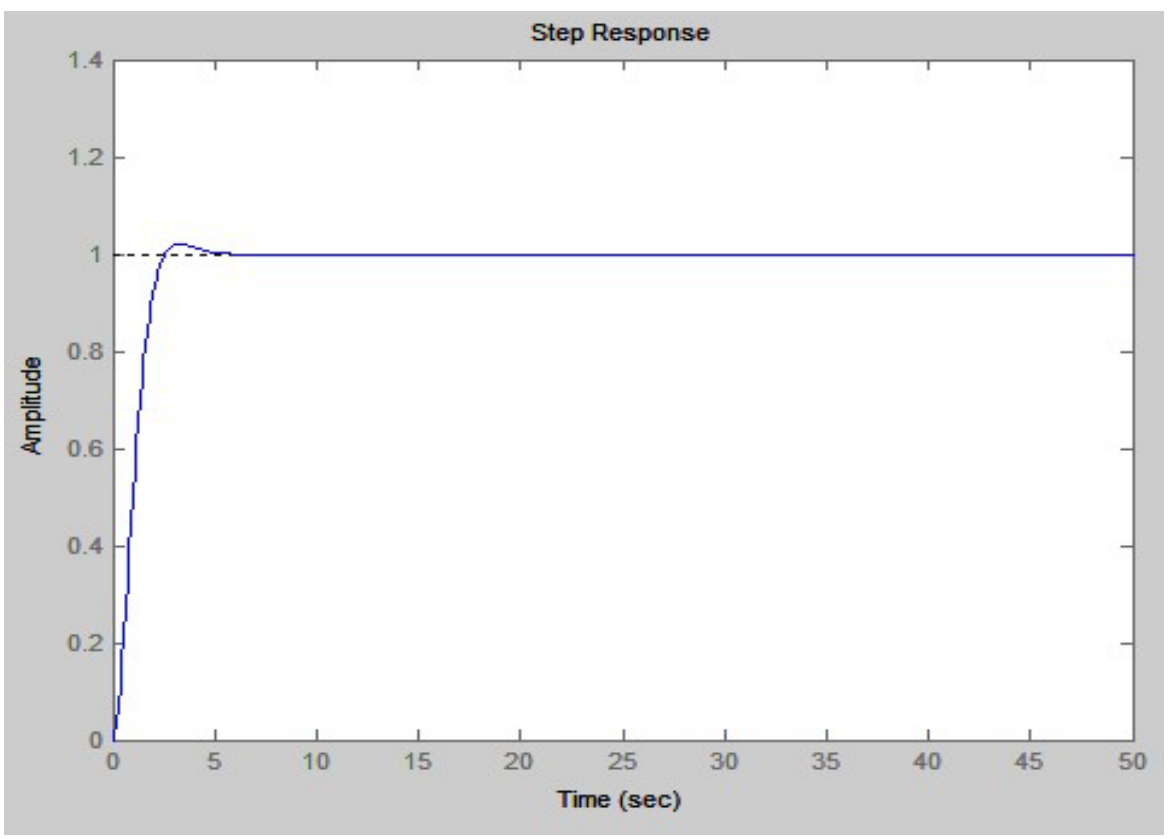

Figure 8. Time response of perturbed plant with TS-based controller

To validate the results the robustness analysis has been carried out the TS- based PID controller was implemented in the perturbed plant as given Eq.(17).The designed TS-based controller given in Eq. (16), were implemented to control the perturbed plant Eq. (17). OThe time domain analysis results depicted in Fig.8, validates that TS-based controller has shown very good robustness performance

$$
G_{\Delta}(s)=\frac{551.1 e^{-0.12 s}}{\left(8 s^{2}+43.26 s+536.9\right)}
$$

\section{Conclusions \& Recommendation}

An assumed weight function $W_{l}$ that satisfy the specifications have been evaluated by TS, an adequate weight function selection, the performance of the designed TS based controller is better than the responses from the PID tuned by $H_{\infty}$ and $\mathrm{ZN}$. The system performance has been analyzed; robust stability conditions of the designed system satisfy the problem. TS is adopted to solve this problem and to achieve the control parameters of the proposed controller. The obtained results have higher fitness and faster convergence.

Though there are several methodologies for PID tuning; conversely, our recommended procedure is a substitute which considers directly the performance conditions and robustness in the design. Furthermore, in the suggested method, the controller structure is not restricted to PID. The proposed procedures could still be applied functionally to any fixed-structure controller.

\section{Figure 8. Time response of perturbed plant with TS-based controller}




\section{REFERENCES}

[1] P. Shubham, K. Meenakshi and G. Rajeev, Application of Artificial Bee Colony Optimization for optimal PID tuning, Advances in Engineering and Technology Research (ICAETR), 2014 International Conference on, Unnao, 2014, pp. 1-5.

[2] F. Mahar, S. Saad Azhar Ali and A. Karim, "Design of fixed order robust controller using $\mathrm{H} \infty$ norm and evolutionary techniques: comparison and performance analysis, Journal of Engineering and Applied Sciences (JEAS), Vol.29 No.1, pp.131-140 Jan-June, 2010

[3] Y. Chaudhari, design and implementation of intelligent controller for a continuous stirred tank reactor system using genetic algorithm, International Journal of Advances in Engineering \& Technology, Mar. 2013, vol. 6, issue1. pp.325-335

[4] F. Glover, and M. Laguna, 1997. Tabu Search. Norwell, MA: Kluwer Academic Publishers.

[5] S. Hanafi, 2001. On the Convergence of Tabu Search. Journal of Heuristics. Vol. 7, pp. $47-58$.

[6] Hertz, A., Taillard, E. and Werra, D. A Tutorial on Tabu Search. Accessed on April 14, 2005

[7] F.S Hillier,. and G.J Lieberman,. Introduction to Operations Research. New York, NY: McGraw-Hill. 8th Ed. 2005.

[8] D.T Pham,. and D Karaboga,. Intelligent Optimization Techniques - Genetic Algorithms, Tabu Search, Simulated Annealing and Neural Networks. London: Springer-Verlag. 2000.

[9] F. Mahar and A. A. Saad \& K. Abid, Design of fixed order robust controller by using evolutionary optimization techniques: Comparison and Performance Analysis, journal of engineering and applied science. vol.29, 2010131-139

[10] Y. Mitsukura, T. Yamamoto \& M. Kaneda, A design of self-tuning PID controllers using a genetic algorithm, Proc. Amer. Contr. Conf., San Diego, CA, 1999, 1361-1365.

[11] Ji, M. and Tang, H.Globa,1 Optimizations and Tabu Search Based on Mamory. Applied Mathematics and Computation. Vol. 159, pp. 449 - 457, 2004.

[12] D.T Pham,. and D Karaboga,. Intelligent Optimization Techniques - Genetic Algorithms, Tabu Search, Simulated Annealing and Neural Networks. London: Springer-Verlag. 2000 .

[13] Reeves, 1993. Modern Heuristic Techniques for Combinatorial Problems. John Wiley \& Sons, Inc.

[14] Zhou, K. Doyle, J. C. and Glover, K., "Robust and Optimal Control", Prentice Hall, New Jersey, 1996.

[15] A. Christiansson, and B. Lennartson, "Weight selections for $H_{\infty}$ control using Genetic Algorithms", Proceedings of the Triennial World Congress Beijing Chin, pp.25-30, 1999.

[16] K. Zhou, and J. C. Doyle, "Essentials of Robust Control," Prentice Hall, Upper Saddle River, New Jersey. 1998. 